Journal of Southeast Asian

Volume 15

Issue 2 Voices from the Field: Centering

Southeast Asian Americans through Policy,

Article 9

Practice, and Activism

2020

\title{
Open Letter to Community: A Call for Unity and Solidarity in the Face of Violence
}

Coalition of Asian American Leaders Minnesota

Coalition of Asian American Leaders Minnesota, info@caalmn.org

Follow this and additional works at: https://docs.lib.purdue.edu/jsaaea

Part of the Bilingual, Multilingual, and Multicultural Education Commons

\section{Recommended Citation}

Minnesota, Coalition of Asian American Leaders (2020) "Open Letter to Community: A Call for Unity and Solidarity in the Face of Violence," Journal of Southeast Asian American Education and Advancement. Vol. 15 : Iss. 2, Article 9.

DOI: $10.7771 / 2153-8999.1213$

Available at: https://docs.lib.purdue.edu/jsaaea/vol15/iss2/9

This document has been made available through Purdue e-Pubs, a service of the Purdue University Libraries.

Please contact epubs@purdue.edu for additional information.

This is an Open Access journal. This means that it uses a funding model that does not charge readers or their institutions for access. Readers may freely read, download, copy, distribute, print, search, or link to the full texts of articles. This journal is covered under the CC BY-NC-ND license. 


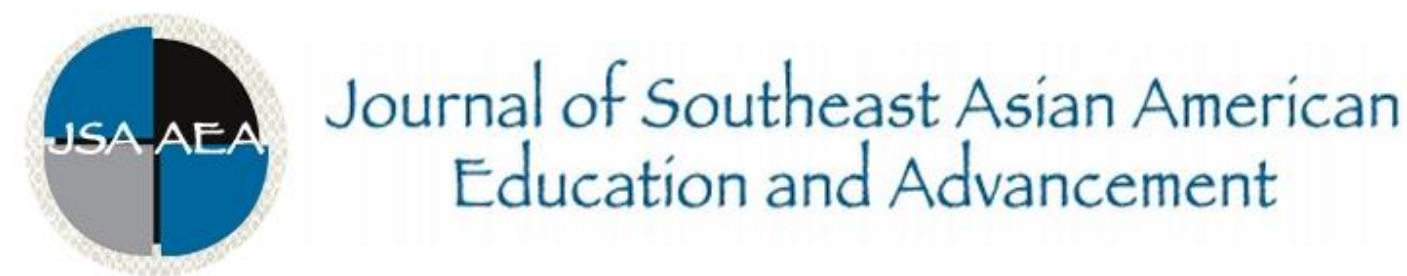

Vol. 15 Iss. 2 Special Issue (2020) www.JSAAEA.org

\author{
Open Letter to Community: \\ A Call for Unity and Solidarity in the Face of Violence
}

\author{
Coalition of Asian American Leaders Minnesota (CAALMN)
}

May 29, 2020

Dear Community,

Earlier this week, George Floyd, a 46-year-old Black man, was murdered by the Minneapolis police. George Floyd's death is a continuation of the long history of criminalization, dehumanization, and oppression of Black lives in this country since its founding. As immigrants and refugees to the United States, our families may not always understand this history, but we inherited its legacy. Our communities have also benefited from Black freedom struggles that paved the way for our own fights for freedom and equal treatment in America. So, in this moment, it matters that we commit to Black liberation and raise our voices to say that \#BlackLivesMatter. We emphatically call on our Asian communities to center George Floyd's life and continue to amplify the demands from his family and community for justice.

Already, Black, Indigenous, and people of color communities (BIPOC) in Minnesota were disproportionately suffering from the COVID-19 pandemic. Our families and small businesses are struggling, the health impacts on communities of color have been disproportionately high, our family members are disproportionately on the front lines in essential jobs, and racist and xenophobic stigmatization early on resulted in the increase of anti-Asian violence. So, we write this knowing that BIPOC communities are hurting badly from the pandemic. Now, as Asian community members are targeted and businesses are damaged, our communities are in pain. We recognize that pain will continue to be there for Asian communities. Yet as we care for each other, we cannot let our pain distract us from George Floyd's life and the community's demands for justice.

We also cannot ignore the role of Officer Tou Thao who stood watch as George Floyd was dying. To see someone who looks like us behave as a bystander to Black death is devastating and painful. This is yet another reason that we must recognize our silence in the face of anti-Black racism, and commit to the ongoing work to dismantle anti-Blackness.

\footnotetext{
@)

SDRERIIGHISRESERNEDReaders are free to copy, display, and distribute this article, as long as the work is attributed to the author(s) and the Journal of Southeast Asian American Education \& Advancement, it is distributed for non-commercial purposes only, and no alteration or transformation is made in the work. More details of this Creative Commons license are available at http://creativecommons.org/licenses/by-nc-nd/3.0/. All other uses must be approved by the author(s) or JSAAEA.

Journal of Southeast Asian American Education \& Advancement, Vol. 15. Iss. 2. (2020) ISSN: 2153-8999
} 
Throughout history, there have been attempts to pit Asian and Black communities against each other, a tactic that encourages us to turn on each other rather than tackle our common oppression: the systems of white supremacy. These efforts distract us from the real solution of building crossracial solidarity to root out racist oppression. And while Asian communities have been rewarded for our assimilation into whiteness with the lie of the "model minority" myth, it is at times like this crisis that we should remember that our status is always conditional and subject to being taken away by xenophobia.

That is why in this painful moment, we ask our Asian communities to choose our shared liberation. Let us remain focused on the systems responsible for the loss of George Floyd's life and too many other Black lives, most recently including Tony McDade, Breonna Taylor, and Ahmaud Arbery. Let us support and uplift Black-led organizing happening through NAACP Mpls, Black Visions Collective, Black Immigrant Collective, Black Lives Matter MN, Reclaim the Block and MN Freedom Fund. We amplify their demands to Minneapolis City Council Members, Mayor Frey, County Attorney Freeman, and other authorities to charge all of the officers involved and to divest from the MPD and other systems of oppression.

Let us stand united for Black lives not only when lives are lost, but in everyday recognition that our liberation is tied together. Let us also commit to the ongoing work of addressing the antiBlackness in our own communities and choose to fight for Black lives the way we would our own. Our struggles must be linked to truly achieve our vision of a Minnesota and a country where all our communities can thrive.

In solidarity,

\section{Asian Minnesotan Organizations}

- Alliance of Progressive South Asians (APSA)

- ArtCrop

- Asian American Organizing Project (AAOP)

- Asian Pacific American Labor Alliance-MN (APALA-MN)

- CAPI USA

- Coalition of Asian American Leaders (CAAL)

- Cultural Society of Filipino Americans (CSFA)

- Families Together

- Filipinx for Immigrant Rights and Racial justice MN (FIRM)

- Green Card Voices

- Hmong American Partnership (HAP)

- India Association of Minnesota (IAM)

- Japanese American Citizens League, Twin Cities Chapter (TC JACL)

- Lao Assistance Center of MN (LACM)

- ManForward

- Minnesota Asian Pacific American Bar Association (MNAPABA)

- Minnesota Hmong Chamber of Commerce

- National Asian Pacific American women's Forum (NAPAWF) - St. Cloud Chapter

- National Association of Asian American Professionals - Minnesota (NAAAP MN) 
- Karen Organization of Minnesota

- Pan Asian Voice for Equity-MN (PAVE-MN)

- Philippine Study Group of Minnesota (PSGM)

- Release MN8

- Reviving the Islamic Sisterhood for Empowerment (RISE)

- Sewa-Aifw

- SEIU Asian Pacific Islander Caucus (SEIU-API)

- Siengkane Lao MN

- TaikoArts Midwest

- Tibetan National Congress

- Theater Mu

- The Southeast Asian Diaspora (SEAD) Project

- Transforming Generations

- Twin Cities South Asia Solidarity Collective

- United Senior Lao-American

- UMN Asian American Studies Program

- UMN Asian American Pacific Islander Desi Faculty \& Staff Association (AAPID FSA)

- Vietnamese Social Services of Minnesota

\section{Out of State Organizations in Solidarity}

- $\$ 6.99$ Per Pound Podcast

- 18 Million Rising

- 1990 Institute

- AAPIs for Civic Empowerment-EF

- Act To Change

- Adhikaar

- Adoptees for Justice

- Alliance of South Asians Taking Action (ASATA)

- American Center of Philippine Arts

- Angel Island Immigration Station Foundation

- APAPA-TX

- Apex Express

- API Community Leaders of Oregon

- API Middle Tennessee

- Apna Ghar, Inc.

- Ascend - Greater Washington

- Asia Pacific Cultural Center

- Asian \& Latino Solidarity Alliance of Central Virginia

- Asian \& Pacific Islander Alliance at Butler University

- Asian \& Pacific Islander American Health Forum

- Asian American Advocacy Fund

- Asian American Arts Alliance

- Asian American Documentary Network (A-Doc)

- Asian American Educators of Howard County

- Asian American Federation 
- Asian American Justice + Innovation Lab (AAJIL)

- Asian American LEAD

- Asian American Legal Defense and Education Fund (AALDEF)

- Asian American Pacific Islander Coalition

- Asian American Psychological Association

- Asian American Psychological Association Division on Filipinx Americans

- Asian American Psychological Association Division on Women

- Asian American Unity Coalition (AAUC)

- Asian American Women's Political Initiative

- Asian American Writers' Workshop

- Asian Americans Advancing Justice

- Asian Americans Advancing Justice - Chicago

- Asian Americans Advancing Justice - Los Angeles

- Asian Americans Advancing Justice - Atlanta

- Asian Americans for Community Involvement

- Asian Americans in Action

- Asian Americans United

- Asian Americans/Pacific Islanders in Philanthropy

- Asian and Pacific Islander American Vote (APIAVote)

- Asian and Pacific Islander Queers United for Action (AQUA)

- Asian Business Association

- Asian CineVision

- Asian Community \& Cultural Center

- Asian Community Development Corporation

- Asian Community Development Council (ACDC)

- Asian Counseling and Referral Service

- Asian Faculty \& Staff Association - University of Wisconsin-Milwaukee

- Asian Law Alliance

- Asian Pacific American Democratic Caucus of Alameda County (California)

- Asian Pacific American Institute for Congressional Studies (APAICS)

- Asian Pacific American Labor Alliance, AFL-CIO

- Asian Pacific American Law Student Association, Northeastern University School of Law (NUSL APALSA)

- Asian Pacific American Media Coalition

- Asian Pacific American Medical Student Association

- Asian Pacific American Network of Oregon (APANO)

- Asian Pacific Community in Action

- Asian Pacific Environmental Network

- Asian Pacific Island Council of San Francisco (API Council)

- Asian Pacific Islander Civic Engagement (ACE) Collaborative

- Asian Pacific Islander Coalition - Spokane Chapter

- Asian Pacific Islander Coalition of South Puget Sound (APIC-SPS)

- Asian Pacific Islander Coalition Pierce County

- Asian Pacific Islander Cultural Center (SF)

- Asian Pacific Islander Faculty \& Staff Association, California State University East Bay

- Asian Pacific Islander Legal Outreach 
- Asian Pacific Islander Small Business Program

- Asian Pacific Partners for Empowerment, Advocacy and Leadership (APPEAL)

- Asian Pacific Policy and Planning Council

- Asian Pasifika Arts Collective

- Asian Podcast Network

- Asian Professional Exchange

- Asian Real Estate Association of America

- Asian Services in Action

- Asian Student Association (Southwestern University)

- Asian Task Force Against Domestic Violence - Massachusetts

- Asian Women for Health

- Asian Women Giving Circle

- Asian/Pacific Islander Domestic Violence Resource Project

- Asians4BlackLives

- Association of Asian Pacific Community Health Organizations

- Austin Asian Complete Count Committee

- Austin Pune Sister City International

- Baltimore Asian Solidarity in Resistance (BARS)

- Banyan: Asian American Writers Collective

- Bay Area Against Hindu Fascism

- BAYAN USA Northeast

- Boston Korean Adoptees

- Brookline Asian American Family Network

- CA Heathy Nail Salon Collaborative

- CAAAV: Organizing Asian Communities

- Cambodia Town, Inc.

- Cambodian American Girls Empowering

- Cambodian Coordinating Council

- Cambodian Mutual Assistance Association of Greater Lowell (CMAA)

- Coalition of Asian Pacifics in Entertainment (CAPE)

- Center for Asian American Media

- Center for Asian American Theology and Ministry at Fuller Theological Seminary

- Center for Empowered Politics

- Cherry Blossom Giving Circle

- Chhaya CDC

- Chinatown Community Development Center

- Chinatown Community Land Trust

- Chinese American Citizens Alliance

- Chinese American Citizens Alliance/DC

- Chinese American Museum of Chicago

- Chinese American Planning Council (CPC)

- Chinese American Progressive Action

- Chinese American Service League

- Chinese American Voices

- Chinese Culture Center of San Francisco

- Chinese Culture Connection, Inc. 
- Chinese for Affirmative Action

- Chinese Progressive Association

- Chinese Progressive Association - Boston

- Chinese Progressive Association - New York City

- Chinese Railroad Workers Descendants Association

- Civic Leadership USA

- Coalition Against Fascism in India

- Coalition for a Better Chinese American Community

- Coalition of Asian Pacific Americans (CAPA)

- Community Youth Center of San Francisco

- Conference on Asian Pacific American Leadership (CAPAL)

- Council of Korean Americans

- Daya Inc

- Dear Asian Americans Podcast

- Diasporic Vietnamese Artists Network (DVAN)

- Divided Families USA

- East Bay Asian Local Development Corporation (EBALDC)

- East Coast Asian American Student Union (ECAASU)

- East West Players

- East Wind Ezine

- EMBARC

- Empowering Pacific Islander Communities (EPIC)

- ENLACE New Mexico

- Equality Labs

- Faith United Methodist Church of Torrance, CA

- Filipino Advocates for Justice

- Filipino American Association of Pittsburgh

- Filipino Cultural Association of George Mason University

- Filipino Migrant Center

- Filipino Young Professionals of DC

- Filipinx Artists of Houston

- Formerly Incarcerated Group Healing Together (F.I.G.H.T)

- Friends of India

- Garrett-Evangelical Theological Seminary

- Global THAI Citizen

- Grassroots Asians Rising

- Greater Boston Legal Services Asian Outreach Unit

- Greater Malden Asian American Community Coalition

- Ha:n UMC (United Methodist Church)

- HANA Center

- Harvard Khmer Student Organization

- Hella Heart Oakland

- Helping Link/Mot Dau Noi

- Hindus for Human Rights

- Hmong American Leadership \& Economic Development (HALED)

- Hmong Innovating Politics 
- HomeSight

- Immigrant Social Services (ISS)

- Indian American Coalition of Texas

- Indian Horizon of Florida

- InterIm Community Development Association

- IU HoosierRaas

- Japanese American Citizens League

- Japanese American Citizens League - Wisconsin Chapter

- Japanese American Citizens League - Seattle chapter

- Japanese American National Museum

- Japanese American Cultural \& Community Center

- Japanese Cultural and Community Center of Northern California

- Joint Council of Librarians of Color Inc.

- Khmer Anti-deportation Advocacy Group of WA

- Khmer Girls in Action

- KhushATX

- Korean Adoptees of Chicago

- Korean American Bar Association of San Diego

- Korean American Center

- Korean American Coalition (KAC)

- Korean American Coalition - Chicago

- Korean American Coalition - Oregon

- Korean American Coalition - San Diego

- Korean American Grassroots Conference

- Korean American Scholarship Foundation

- Korean Resource Center

- Laotian American National Alliance (LANA)

- Leadership Education for Asian Pacifics (LEAP)

- Little Tokyo Service Center

- Malden Community Organizing for Racial Equity

- Massachusetts Asian American Educators Association

- Masto Foundation

- Mekong NYC

- Michigan Asian Pacific American Bar Association

- Milwaukee Consortium for Hmong Health, Inc.

- Mindhatch

- MinKwon Center for Community Action

- Model Majority

- NAKASEC Virginia

- National Asian American Pacific Islander Mental Health Association

- National Asian Pacific American Families Against Substance Abuse

- National Asian Pacific American Women's Forum (NAPAWF)

- National Asian Pacific American Women's Forum (NAPAWF) - NYC Chapter

- National Asian Pacific American Women's Forum (NAPAWF) - Washington DC Chapter

- National Asian Pacific Center on Aging (NAPCA) 
- National Association of Asian American Professionals (NAAAP)

- National Association of Asian American Professionals - Atlanta

- National Association of Asian American Professionals - Chicago

- National Association of Asian American Professionals - Colorado

- National Association of Asian American Professionals - Los Angeles

- National Association of Asian American Professionals - Orange County, CA

- National Association of Asian American Professionals - San Diego

- National Association of Asian American Professionals - Seattle

- National Association of Asian American Professionals - Cincinnati

- National Association of Asian American Professionals - Dallas - Fort Worth

- National Association of Asian American Professionals - Washington, D.C.

- National Association of Filipino American Associations Region V

- National CAPACD

- National Federation of Filipino American Associations (NaFFAA)

- National Iranian American Council

- National Korean American Service \& Education Consortium (NAKASEC)

- National Queer Asian Pacific Islander Alliance

- Nepalese American Forum for Political Empowerment-NAFPE

- New Mexico Asian Family Center

- New York Day of Remembrance Committee

- North Carolina Asian Americans Together (NCAAT)

- O-Musubi

- OCA - Asian Pacific American Advocates

- OCA - Asian Pacific American Advocates - Colorado

- OCA - Asian Pacific American Advocates - Greater Chicago

- OCA - Asian Pacific American Advocates - Greater Cleveland

- OCA - Asian Pacific American Advocates - Greater Houston

- OCA - Asian Pacific American Advocates - Greater Seattle Chapter

- OCA - Asian Pacific American Advocates - San Francisco Chapter

- OCA - Asian Pacific American Advocates - Westchester \& Hudson Valley

- OCA - Asian Pacific American Advocates - Wisconsin Chapter

- Ohio AAPI Democratic Caucus

- Ohio Asian American Health Coalition

- OneAmerica

- OPAWL - Building AAPI Feminist Leadership in Ohio

- Orange County Asian and Pacific Islander Community Alliance (OCAPICA)

- Oregon Chinese Consolidated Benevolent Association

- PACE-ECE

- Pacific Asian Counseling Services

- Parivar Bay Area

- Philly's Radical Asian American Womxn's Collective (RAAWC) Core

- Port of Portland Asian Pacific Islander Employee Resource Group

- Providence Youth Student Movement (PrYSM)

- Q-Wave - Building Queer \& Trans API Community since 2004

- Raha Iranian Feminist Collective

- Raksha, Inc. 
- Refugee and Immigrant Services Northwest

- Revolutionary Love Project

- Rising Voices of Asian American Families

- SAAVETX South Asian American Voter Empowerment

- Sadhana: Coalition of Progressive Hindus

- SAG-AFTRA Asian Pacific American Media (APAM) Committee

- SAHI

- Sakhi for South Asian Women

- Sama Sama Cooperative (California Bay Area)

- Samakkee Thai American Coalition

- San Diego Yokohama Sister Cities Society

- Seattle Chinatown-International District Preservation and Development Authority

- Seeding Change

- SF Love Dojo

- Shetu Inc.

- Sikh American Legal Defense and Education Fund

- Sikh Coalition

- Snohomish APIC

- SolidarityIs

- South Asian American Policy and Research Institute (SAAPRI)

- South Asian Americans Leading Together (SAALT)

- South Asian Network

- South Asian Public Health Association (SAPHA)

- South Asian Rapid Response Initiative - Justice for Muslims Collective

- South Asian Workers Center-Boston

- South Asian Youth Action

- South Asians 4 Black Lives

- South Asians in Sports

- Southeast Asia Resource Action Center

- Southeast Asian American Education Coalition

- Southeast Asian Coalition of Central Massachusetts

- Southeast Asian Community Alliance (SEACA)

- Southeast Asian Defense Project

- Stand With Kashmir

- Thai American Bar Association

- Thai and Southeast Asian Association of California

- Thai Community Development Center

- Thammasat University Alumni Association

- The 2020 Project

- The Bulosan Center for Filipino Studies

- The Center for Asian Pacific American Women (CAPAW)

- The Korean American Adoptee Adoptive Family Network (KAAN)

- The National Council of Asian Pacific Americans (NCAPA)

- Tsuru for Solidarity

- UCLA Asian American Studies Center (LA)

- Union of North American Vietnamese Student Associations (UNAVSA) 
- United Cambodian Community

- Venerable-Counselors at Law, PC

- VietLead

- Vietnamese American Initiative for Development

- Visual Communications Media

- VN TeamWork, Inc.

- Womankind

- Women for Afghan Women

- Woori Center

- Yemeni American Merchants Association

Note: This open letter is reprinted with permission. The original statement can be found at https://caalmn.org/2020/05/29/api4georgefloyd/ 


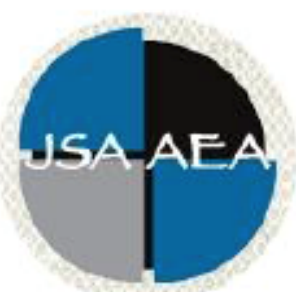

\title{
Journal of Southeast Asian American \\ Education and Advancement
}

Vol. 15 Iss. 2 Special Issue (2020) www.JSAAEA.org

Special Issue Co-Editors

Dr. Peter T. Keo

Stanford University

Dr. Loan Thi Dao

St. Mary's College of California

Editor

Dr. Wayne E. Wright

Purdue University

Associate Editors

Dr. Chhany Sak-Humphry

University of Hawaii at Manoa

Dr. Phitsamay Sychitkokhong Uy

University of Massachusetts, Lowell

\author{
Book Review Editor \\ Dr. Vichet Chhuon \\ University of Minnesota \\ Creative Works Editor \\ Bryan Thao Worra \\ Lao Assistance Center \\ Journal Manager \\ Fang Gao \\ Purdue University
}

\section{Editorial Review Board}

Dr. Steve Arounsack

California State University, Stanislaus

Dr. Sovicheth Boun

Salem State University
Dr. Carl L. Bankston III

Tulane University

Dr. Phala Chea

Lowell Public Schools 


\author{
Dr. Virak Chan \\ Purdue University \\ Dr. Loan Dao \\ St. Mary's College of California \\ Dr. Changming Duan \\ University of Missouri-Kansas City \\ Dr. Sothy Eng \\ Lehigh University \\ Dr. Vincent K. Her \\ University of Wisconsin, Eau Claire \\ Dr. Peter Nien-Chu Kiang \\ University of Massachusetts, Boston \\ Dr. Kevin K. Kumashiro \\ University of Illinois, Chicago \\ Dr. Ha Lam \\ Independent Scholar \\ Dr. Jonathan H. X. Lee \\ San Francisco State University \\ Dr. Monirith Ly \\ Royal University of Phnom Penh \\ Dr. Bic Ngo \\ University of Minnesota \\ Dr. Leakhena Nou \\ California State University, Long Beach \\ Dr. Mark Pfeifer \\ SUNY Institute of Technology \\ Dr. Loan T. Phan \\ University of New Hampshire \\ Dr. Karen Quintiliani \\ California State University, Long Beach \\ Dr. Angela Reyes \\ Hunter College \\ The City University of New York \\ Dr. Fay Shin \\ California State University, Long Beach \\ Dr. Christine Su \\ College of San Mateo \\ Dr. Alisia Tran \\ Arizona State University \\ Dr. Khatharya Um \\ University of California, Berkeley \\ Dr. Kim Tran \\ University of California, Los Angeles, \\ Glendale Community College \\ Dr. Molly Wiebie \\ The University of Texas at Austin
}

Dr. George Chigas

University of Massachusetts, Lowell

Dr. Hien Duc Do

San Jose State University

Dr. Sophal Ear

Occidental College

Dr. Jeremy Hein

University of Wisconsin, Eau Claire

Dr. Nancy H. Hornberger

University of Pennsylvania

Dr. Peter Tan Keo

Stanford University

Dr. Yvonne Kwan

San Jose State University

Dr. Ravy Lao

California State University, Los Angeles

Dr. Stacey Lee

University of Wisconsin, Madison

Dr. Sue Needham

California State University, Dominguez Hills

Dr. Max Niedzwiecki

Daylight Consulting Group

Dr. Clara Park

California State University, Northridge

Dr. Giang Pham

University of Massachusetts Amherst

Dr. Malaphone Phommasa

University of Clifornia Santa Barbara

Dr. Kalyani Rai

University of Wisconsin-Milwaukee

Dr. Cathy J. Schlund-Vials

University of Connecticut, Storrs

Dr. Nancy J. Smith-Hefner

Boston University

Dr. Yer J. Thao

Portland State University

Dr. Monica M. Trieu

Purdue University

Dr. Silvy Un

Saint Paul Public Schools

Dr. Linda Trinh Vo

University of California, Irvine

Dr. Yang Sao Xiong

The University of Wisconsin-Madison

Dr. Zha Blong Xiong

University of Minnesota 


\section{Doctoral Student Editorial Review Board}

Diana Chandara

University of Minnesota-TwinCiteis

Linh Dang

University of Rochester

Annie BichLoan Duong

San Joaquin County Office of Education

Jacqueline Mac

Indiana University

Vanessa Sovanika Na

University of California SanDiego

Khoi Nguyen

George Mason University

Linda Marie Pheng

University of Wisconsin-Madison

Latana Thaviseth

University of California Los Angeles

Melissa Vang

San Diego State University

Soua Xiong

San Diego State University

Claremont Graduate University
Kassandra Chhay

University of Minnesota-Twin Cities

Bao Diep

University of Minnesota-Twin Cities

Nielson Hul

Cornell University

Dung Minh Mao

University of Minnesota-Twin Cities

Hoa Nha Nguyen

Boston College

Thien-Huong Ninh

University of Southern California

Krissyvan Truong

Claremont Graduate University

Mai Vang

University of Massachusetts Boston

Thong Vang

University of Minnesota-Twin Cities 